TRI-PP-94-64

\title{
Field transformations and the classical equation of motion in chiral perturbation theory
}

\author{
S. Scherer* and H. W. Fearing \\ TRIUMF, 4004 Wesbrook Mall, Vancouver, B. C., \\ Canada V6T 2A3
}

(August 15, 1994)

\begin{abstract}
The construction of effective Lagrangians commonly involves the application of the 'classical equation of motion' to eliminate redundant structures and thus generate the minimal number of independent terms. We investigate this procedure in the framework of chiral perturbation theory. The use of the 'classical equation of motion' is interpreted in terms of field transformations. Such an interpretation is crucial if one wants to bring a given Lagrangian into a canonical form with a minimal number of terms. We emphasize that the application of field transformations not only eliminates structures, or, what is equivalent, expresses certain structures in terms of already known different structures, but also leads to a modification of coefficients of higher-order terms. This will become relevant, once one considers effective interaction terms beyond next-to-leading order, i.e., beyond $O\left(p^{4}\right)$.
\end{abstract}

11.30.Rd, 12.39.Fe

Typeset using REVTEX

*Address after Sept. 1, 1994: Institut für Kernphysik, Johannes Gutenberg-Universität, J. J. Becher-Weg 45, D-55099 Mainz, Germany. 


\section{INTRODUCTION}

Effective field theory calculations have been used in different areas of particle physics. Applications include chiral perturbation theory [1-3] where the approximate chiral symmetry of the underlying $Q C D$ Lagrangian is mapped into a systematic momentum expansion of the interaction between the low-energy degrees of freedom, namely the Goldstone bosons of spontaneous chiral symmetry breaking. An extension of chiral perturbation theory which includes the low-lying baryon states has been performed by several authors [4 [0]. Another example involves the treatment of the weak interaction in processes where the external momenta are much smaller than the mass of the $W$ boson (see Ref. [7] and references therein). Under these circumstances S-matrix elements may be expanded in terms of the ratio $p^{2} / M_{W}^{2}$ and an effective Lagrangian approach is convenient to represent such an expansion. A further application is concerned with the effects on observables at presently available energies of heavy particles beyond the standard model [8,99].

All the above examples have in common that at a certain point in the construction of the most general structures of the effective Lagrangian use of the 'classical equation of motion' is made in order to eliminate redundant structures (see, e.g., Refs. [2, 3, 10, 11]). A thorough discussion on the formal aspects including, in particular, the relation between the 'classical equation of motion' and field transformations can be found in Refs. [7, 9, 12, 13]. The basic motivation for the use of field transformation is the equivalence theorem which states that observables should not depend on the specific choice for the interpolating field [14 I6].

In this work we concentrate on practical aspects of applying field transformations in the framework of chiral perturbation theory. Concerning the formal justification of using field transformations in effective theories we refer the reader to Refs. 99.13. Firstly, we identify the relevant properties of field transformations for the specific case of chiral perturbation theory. Then we make contact with the use of the 'classical equation of motion' as a means to eliminate redundant structures in the construction of the most general chiral Lagrangian. Part of this discussion has already been outlined in Ref. [12].

Secondly, we address the question how to bring a given effective Lagrangian into a canonical form, i.e., a form with the minimal number of independent terms. This issue is of practical importance since an effective Lagrangian obtained from, say, the bosonization of an effective quark model action, is in general not in a canonical form. In order to allow for an easy comparison between the predictions of different models it is most convenient to bring the effective Lagrangian into a generally accepted canonical form. For example, at $O\left(p^{4}\right)$ the commonly used standard is the one by Gasser and Leutwyler [3].

The formal procedure turns out to be very similar to the Foldy-Wouthuysen transformation [17,18 where a sequence of transformations is applied in order to bring a Dirac Hamiltonian into a block-diagonal form up to a given order in the $1 / M$ expansion. The analogy in chiral perturbation theory is a sequence of transformations which removes redundant terms up to a given order in the momentum expansion. In both methods it is crucial to consistently keep all terms up to and including the desired final order at each step in the iteration process. 


\section{THE CHIRAL LAGRANGIAN AND FIELD TRANSFORMATIONS}

In the momentum expansion a chiral Lagrangian is written as a sum of terms with an increasing number of covariant derivatives, external fields (including quark mass terms), and field strength tensors,

$$
\tilde{\mathcal{L}}=\mathcal{L}_{2}+\tilde{\mathcal{L}}_{4}+\tilde{\mathcal{L}}_{6}+\ldots
$$

For the moment, we assume that the chiral Lagrangian is not yet in a canonical form, which we define to be a form with a minimal number of independent structures at a given order. We denote a non-canonical form by $\tilde{\mathcal{L}}$. The lowest-order term in Eq. (2.1) is, up to a total derivative, unique [3] and we may thus omit the tilde,

$$
\mathcal{L}_{2}(U)=\frac{F_{0}^{2}}{4} \operatorname{Tr}\left(D_{\mu} U\left(D^{\mu} U\right)^{\dagger}\right)+\frac{F_{0}^{2}}{4} \operatorname{Tr}\left(\chi U^{\dagger}+U \chi^{\dagger}\right), \quad U(x)=\exp \left(i \frac{\phi(x)}{F_{0}}\right),
$$

where $\phi(x)$ is a traceless, hermitian $3 \times 3$ matrix containing the eight Goldstone bosons $(\pi, K, \eta), D_{\mu}$ is a covariant derivative, $F_{0}$ is the pion decay constant in the chiral limit, and $\chi$, eventually, contains the quark masses (for details see Ref. [3]). The most general canonical Lagrangian at $O\left(p^{4}\right), \mathcal{L}_{4}^{G L}$, was obtained by Gasser and Leutwyler [3]. Use of the 'classical equation of motion', i.e., the one obtained from $\mathcal{L}_{2}$, was made to eliminate two additional structures at $O\left(p^{4}\right)$. In the following we will interpret this procedure in terms of field transformations [8,12,15,16].

Suppose we want to perform a field transformation where we demand the same properties of another 'interpolating field' $U^{\prime}(x)$ as of $U(x)$, namely, both are $S U(3)$ matrices and transform under the group $\left(G=S U(3)_{L} \times S U(3)_{R}\right)$, parity, and charge conjugation, respectively, according to

$$
W \stackrel{G}{\rightarrow} V_{R} W V_{L}^{\dagger}, \quad W(\vec{x}, t) \stackrel{P}{\rightarrow} W^{\dagger}(-\vec{x}, t), \quad W \stackrel{C}{\rightarrow} W^{T},
$$

for $W=U$ or $U^{\prime}$. In Eq. 2.3) $V_{L}$ and $V_{R}$ are independent $S U(3)$ matrices representing group elements of $G$. Since both, $U$ and $U^{\prime}$, are supposed to be $S U(3)$ matrices, they can be related through

$$
U=\exp \left(i S\left(U^{\prime}\right)\right) U^{\prime}
$$

where $S=S^{\dagger}$, and $\operatorname{Tr}(S)=0$. The generator $S$ may, in general, depend on all building blocks which are required for the construction of the chiral Lagrangian. The properties of $U$ and $U^{\prime}$, Eq. (2.3), result in the following requirements for $S$ :

$$
S \stackrel{G}{\rightarrow} V_{R} S V_{R}^{\dagger}, \quad S(\vec{x}, t) \stackrel{P}{\rightarrow}-U^{\prime \dagger}(-\vec{x}, t) S(-\vec{x}, t) U^{\prime}(-\vec{x}, t), \quad S \stackrel{C}{\rightarrow}\left(U^{\prime \dagger} S U^{\prime}\right)^{T} .
$$

For example, two such generators exist at $O\left(p^{2}\right)$ in the momentum expansion (denoted by the subscript 2 of $S)$ 円:

\footnotetext{
${ }^{1}$ When dealing with $U(3)$ matrices, $S_{2}$ does not have to satisfy the constraint $\operatorname{Tr}\left(S_{2}\right)=0$, and thus one obtains $i \alpha_{3} \operatorname{Tr}\left(\chi U^{\prime \dagger}-U^{\prime} \chi^{\dagger}\right)$ as an additional independent generator.
} 


$$
S_{2}\left(U^{\prime}\right)=i \alpha_{1}\left(D^{2} U^{\prime} U^{\prime \dagger}-U^{\prime}\left(D^{2} U^{\prime}\right)^{\dagger}\right)+i \alpha_{2}\left(\chi U^{\prime \dagger}-U^{\prime} \chi^{\dagger}-\frac{1}{3} \operatorname{Tr}\left(\chi U^{\prime \dagger}-U^{\prime} \chi^{\dagger}\right)\right),
$$

where $\alpha_{1}$ and $\alpha_{2}$ are arbitrary real parameters. Note that $S_{2}\left(U^{\prime}\right)$ depends, in addition to $U^{\prime}$ and $U^{\prime \dagger}$, on $D^{2} U^{\prime}, \chi, \ldots$. We are somewhat sloppy since we denote this dependence collectively by the symbol $U^{\prime}$.

Let us now investigate the change in the functional form of $\tilde{\mathcal{L}}$ due to the field transformation. After inserting Eq. (2.4) into Eq. (2.1) the result can be expanded as

$$
\tilde{\mathcal{L}}(U)=\sum_{n=0}^{\infty} \delta^{(n)} \tilde{\mathcal{L}}\left(U^{\prime}, S\right), \quad \delta^{(0)} \tilde{\mathcal{L}}\left(U^{\prime}, S\right) \equiv \tilde{\mathcal{L}}\left(U^{\prime}\right)
$$

where the superscripts denote the power of $\mathrm{S}$ (or $D_{\mu} S, \ldots$ ). Furthermore, each term of Eq. (2.7) may be decomposed according to its origin in the momentum expansion,

$$
\delta^{(n)} \tilde{\mathcal{L}}\left(U^{\prime}, S\right)=\delta^{(n)} \mathcal{L}_{2}\left(U^{\prime}, S\right)+\delta^{(n)} \tilde{\mathcal{L}}_{4}\left(U^{\prime}, S\right)+\ldots
$$

For example, the lowest-order change is given by

$$
\delta^{(1)} \mathcal{L}_{2}\left(U^{\prime}, S\right)=\frac{F_{0}^{2}}{4} \operatorname{Tr}\left(i S \mathcal{O}_{E O M}^{(2)}\left(U^{\prime}\right)\right)
$$

where

$$
\mathcal{O}_{E O M}^{(2)}\left(U^{\prime}\right)=D^{2} U^{\prime} U^{\prime \dagger}-U^{\prime}\left(D^{2} U^{\prime}\right)^{\dagger}-\chi U^{\prime \dagger}+U^{\prime} \chi^{\dagger}+\frac{1}{3} \operatorname{Tr}\left(\chi U^{\prime \dagger}-U^{\prime} \chi^{\dagger}\right) .
$$

In the following we do not assume that Eq. (2.10) is identical to zero, even though it has the functional form of the 'classical equation of motion' derived from Eq. (2.2) [3].

For a generator $S_{2 m}(m \geq 1)$ of $O\left(p^{2 m}\right)$, the expression $\delta^{(n)} \tilde{\mathcal{L}}_{2 k}\left(U^{\prime}, S_{2 m}\right)$ is of $O(2 k+$ $2 m \times n)$ in the momentum expansion. In practical applications one only considers the chiral Lagrangian up to a small order, say $O\left(p^{4}\right)$ or $O\left(p^{6}\right)$. This automatically restricts the construction of relevant generators to $O\left(p^{2}\right)$ or $O\left(p^{2}\right)$ and $O\left(p^{4}\right)$, respectively.

By choosing suitable field transformations it is possible to 'eliminate' structures at $O\left(p^{4}\right)$ and higher in the momentum expansion, and bring $\tilde{\mathcal{L}}$ into a canonical form $\mathcal{L}$. To illustrate this method we first give the most general non-canonical form of the chiral Lagrangian at $O\left(p^{4}\right)$ (prior to the application of the 'classical equation of motion') [19],

$$
\begin{aligned}
\tilde{\mathcal{L}}_{4}(U)= & \mathcal{L}_{4}^{G L}(U)+c_{1} \operatorname{Tr}\left(\left(D^{2} U U^{\dagger}-U\left(D^{2} U\right)^{\dagger}\right) \mathcal{O}_{E O M}^{(2)}\right) \\
& +c_{2} \operatorname{Tr}\left(\left(\chi U^{\dagger}-U \chi^{\dagger}\right) \mathcal{O}_{E O M}^{(2)}\right)
\end{aligned}
$$

\footnotetext{
${ }^{2}$ In the following we deliberately omit total-derivative terms, since they do not modify the equation of motion.

${ }^{3}$ We adhere to the commonly used convention for the classical equation of motion (see Eq. (5.9) of Ref. [3]), even though, from a formal point of view, it is more natural to use the hermitian combination $i \frac{F_{0}^{2}}{4} \mathcal{O}_{E O M}^{(2)}$ obtained from the variation of $\mathcal{L}_{2}$.
} 
where we do not need to present the explicit form of $\mathcal{L}_{4}^{G L}$ of Gasser and Leutwyler for the present purpose. If we insert the field transformation of Eq. (2.4) with $S_{2}$ of Eq. (2.6) into $\mathcal{L}_{2}(U)+\tilde{\mathcal{L}}_{4}(U)$, and choose $\alpha_{1}=4 c_{1} / F_{0}^{2}$ and $\alpha_{2}=4 c_{2} / F_{0}^{2}$, the last two terms of Eq. (2.11) and the modification $\delta^{(1)} \mathcal{L}_{2}$ precisely cancel and one obtains the result of Gasser and Leutwyler (after renaming $U^{\prime}$ into $U$ ). In other words, the Lagrangians $\mathcal{L}_{2}(U)+\mathcal{L}_{4}^{G L}(U)$ and $\mathcal{L}_{2}(U)+\tilde{\mathcal{L}}_{4}(U)$ are equivalent up to $O\left(p^{4}\right)$ and thus, according to the equivalence theorem, should generate the same (on-shell) S-matrix elements at $O\left(p^{4}\right)$. In this sense, namely by identifying structures which are proportional to $\mathcal{O}_{E O M}^{(2)}$, the 'classical equation of motion' can be used to eliminate terms in an effective Lagrangian.

This situation is often interpreted in the following manner (see, e.g., Refs. [10,20]). The equation of motion in the presence of higher-order terms can be written as

$$
\mathcal{O}_{E O M}^{(2)}(U)=0+O\left(p^{4}\right) .
$$

Inserting Eq. (2.12) into Eq. (2.11) and dropping terms which are of $O\left(p^{6}\right)$ generates $\mathcal{L}_{4}^{G L}(U)$. However, even though this argument is intuitively appealing and correct at this order, it also contains a potential source of error, as soon as one tries to apply it in the presence of higher-order structures. We will discuss this point in more detail below.

It is straightforward to extend the use of field transformations to higher-order terms. Suppose the higher-order Lagrangian contains a chirally invariant structure of the type

$$
\begin{aligned}
& \operatorname{Tr}\left(\left(D^{2} U U^{\dagger}-U\left(D^{2} U\right)^{\dagger}\right) A\right)=\operatorname{Tr}\left(\left(D^{2} U U^{\dagger}-U\left(D^{2} U\right)^{\dagger}\right) \bar{A}\right) \\
& =\operatorname{Tr}\left(\mathcal{O}_{E O M}^{(2)} \bar{A}\right)+\operatorname{Tr}\left(A\left(\chi U^{\dagger}-U \chi^{\dagger}\right)\right)-\frac{1}{3} \operatorname{Tr}\left(\chi U^{\dagger}-U \chi^{\dagger}\right) \operatorname{Tr}(A),
\end{aligned}
$$

where $\bar{A}=A-\frac{1}{3} \operatorname{Tr}(A)$ is traceless and of $O\left(p^{4}\right)$ or higher. We used $\operatorname{Tr}\left(D^{2} U U^{\dagger}-U\left(D^{2} U\right)^{\dagger}\right)=$ 0 and $\operatorname{Tr}(\bar{A})=0$ in the first and second step, respectively. With the help of a field transformation involving $\bar{A}$ the first term can be eliminated and the application of the field transformation looks as if one had simply made the replacement

$$
D^{2} U U^{\dagger}-U\left(D^{2} U\right)^{\dagger} \rightarrow \chi U^{\dagger}-U \chi^{\dagger}-\frac{1}{3} \operatorname{Tr}\left(\chi U^{\dagger}-U \chi^{\dagger}\right)
$$

in order to replace the original expression in terms of two structures which one anyway has to considerf.

However, at this point one should carefully distinguish between the following two situations which are of practical interest. The first regards the pure identification of terms necessary for the construction of the most general chiral Lagrangian. For this purpose it is completely sufficient to use the 'classical equation of motion' in the sense of Eq. (2.14). This

\footnotetext{
${ }^{4}$ For convenience we use the same symbol $U$ in both Lagrangians.

${ }^{5}$ At present we cannot exclude the possibility that in some cases it might be advantageous to reverse the direction of the arrow in Eq. (2.14), i.e., to perform the replacement the other way around.
} 
can be understood as follows [8]. Even though a suitable field transformation containing a generator of $O\left(p^{2 m}\right)$ not only removes or reexpresses a term at $O\left(p^{2 m+2}\right)$ but also modifies the interaction terms at $O\left(p^{2 m+4}\right)$ and higher, this modification concerns the coefficients of structures which one anyway has to consider in the construction of the most general Lagrangian. As long as one is not yet interested in the specific values of these coefficients, one can freely make use of Eq. (2.14) to reduce the number of structures.

A different and more complicated situation occurs if one actually wants to bring a given non-canonical form of a chiral Lagrangian into a canonical form. Non-canonical forms typically arise in effective Lagrangians which are obtained after bosonization of an effective $Q C D$ inspired quark interaction [21 24]. For the purpose of comparing different effective Lagrangians it is most convenient to bring them into a commonly accepted standard form and to simply compare the predictions for the coefficients. For example, at $O\left(p^{4}\right)$ it is standard practice to use the canonical form of Gasser and Leutwyler [3].

In the following we will discuss the issue of transforming chiral Lagrangians beyond $O\left(p^{4}\right)$ into a canonical form. For practical purposes we restrict our discussion to terms up to $O\left(p^{6}\right)$. Clearly, an extension to higher orders is straightforward, but very tedious. Suppose one starts with predictions for the coefficients of a Lagrangian $\tilde{\mathcal{L}}=\mathcal{L}_{2}+\tilde{\mathcal{L}}_{4}+\tilde{\mathcal{L}}_{6}$, where $\tilde{\mathcal{L}}_{4}$ is not yet in the form of the Lagrangian of Gasser and Leutwyler. In addition, $\tilde{\mathcal{L}}_{6}$ may not yet be in the canonical form.

A naive application of the equation of motion argument, namely, a replacement according to Eq. (2.14) will not yield the correct result, i.e., it will not generate an equivalent Lagrangian which reproduces the same $\mathrm{S}$-matrix elements as the original Lagrangian. The reason is that even though the replacement using Eq. (2.14) produces the required structures, it does not automatically give the correct coefficients. To be specific, the transformation procedure using $S_{2}$ as described following Eq. (2.11) results in the following Lagrangian at $O\left(p^{6}\right)$

$$
\tilde{\mathcal{L}}_{6}^{\prime}\left(U^{\prime}\right)=\tilde{\mathcal{L}}_{6}\left(U^{\prime}\right)+\delta^{(1)} \tilde{\mathcal{L}}_{4}\left(U^{\prime}, S_{2}\right)+\delta^{(2)} \mathcal{L}_{2}\left(U^{\prime}, S_{2}\right)
$$

where

$$
\begin{aligned}
\delta^{(1)} \tilde{\mathcal{L}}_{4}\left(U^{\prime}, S\right)= & \frac{F_{0}^{2}}{4} \operatorname{Tr}\left(i S \mathcal{O}_{E O M}^{(4)}\left(U^{\prime}\right)\right) \\
\delta^{(2)} \mathcal{L}_{2}\left(U^{\prime}, S\right)= & \frac{F_{0}^{2}}{4} \operatorname{Tr}\left(S\left(D_{\mu} U^{\prime} U^{\prime \dagger} D^{\mu} S-D^{\mu} S D_{\mu} U^{\prime} U^{\prime \dagger}-D^{2} S\right)\right. \\
& \left.-\frac{1}{2}\left(\chi U^{\prime \dagger}+U^{\prime} \chi^{\dagger}\right) S^{2}\right)
\end{aligned}
$$

In Eq. (2.16) $\mathcal{O}_{E O M}^{(4)}$ refers to the contribution to the equation of motion derived from $\tilde{\mathcal{L}}_{4}$. Once again, in order to be consistent with Eq. (2.10) we choose a form for $\mathcal{O}_{E O M}^{(4)}$ which differs by a factor $-4 i / F_{0}^{2}$ from what one would obtain with the variational principle. The covariant derivative of an operator $A$ which transforms under the group as $V_{R} A V_{R}^{\dagger}$ (such as $S)$ is defined as

$$
D_{\mu} A=\partial_{\mu} A-i R_{\mu} A+i A R_{\mu} .
$$

Clearly, the two terms of Eq. (2.16) result in an additional contribution to the Lagrangian at $O\left(p^{6}\right)$ which one would have missed had one simply applied Eq. (2.14) to $\tilde{\mathcal{L}}_{4}$, i.e., had one 
simply dropped the terms proportional to the classical equation of motion. Such a procedure is only correct up to $O\left(p^{4}\right)$. Furthermore, from Eq. (2.16) one can also deduce that even a generalization of Eq. (2.12) taking into account $\mathcal{O}_{E O M}^{(4)}$ resulting from $\tilde{\mathcal{L}}_{4}$,

$$
\mathcal{O}_{E O M}^{(2)}=-\mathcal{O}_{E O M}^{(4)}+O\left(p^{6}\right),
$$

does not yield an equivalent Lagrangian since one misses the contribution of $\delta^{(2)} \mathcal{L}_{2}$. Even though Eq. (2.18) is a straightforward extension of Eq. (2.12), it obviously cannot be used to rewrite structures in $\tilde{\mathcal{L}}_{4}$ with a precision up to and including $O\left(p^{6}\right)$. At first sight this looks surprising as one appears to use the same method as before. However, there is a fundamental difference between applying Eq. (2.12) to Eq. (2.11) and the corresponding replacement using Eq. (2.18). In the first case information of $\mathcal{L}_{2}$ is used in the higher-order term $\tilde{\mathcal{L}}_{4}$, whereas in the second case one tries to put back 'information' derived from $\tilde{\mathcal{L}}_{4}$ into the same term. We will illustrate this point in a simple toy model [7] in the Appendix.

We now describe a systematic procedure which consists of successively applying transformations with generators where the order in the momentum expansion increases with each iteration. Let $2 N$ denote the maximal order of a given non-canonical chiral Lagrangian

$$
\tilde{\mathcal{L}}=\mathcal{L}_{2}+\tilde{\mathcal{L}}_{4}+\ldots+\tilde{\mathcal{L}}_{2 N}
$$

which we want to bring into a canonical form up to the same order. Prior to the first iteration step one brings, by suitably adding and subtracting terms, the $O\left(p^{4}\right)$ Lagrangian $\tilde{\mathcal{L}}_{4}$ into the form of Eq. (2.11) and identifies the coefficients $c_{1}$ and $c_{2}$. The first transformation

$$
U=\exp \left(i S_{2}\left(U^{(1)}\right)\right) U^{(1)}
$$

with $S_{2}$ given in Eq. (2.6) and $\alpha_{1}=4 c_{1} / F_{0}^{2}$ and $\alpha_{2}=4 c_{2} / F_{0}^{2}$, results in an equivalent effective Lagrangian which is canonical up to $O\left(p^{4}\right)$. Before applying the second iteration one has to manipulate the new $\tilde{\mathcal{L}}_{6}^{\prime}$ obtained after the first transformation in such a manner as to obtain as many terms as possible containing $\mathcal{O}_{E O M}^{(2)}$ (see Eq. (2.13)). One then applies the transformation

$$
U^{(1)}=\exp \left(i S_{4}\left(U^{(2)}\right)\right) U^{(2)},
$$

with a suitable choice of $S_{4}$. The resulting Lagrangian is canonical up to $O\left(p^{6}\right)$. Clearly, one has to perform $N-1$ iterations in order to obtain a canonical Lagrangian up to $O\left(p^{2 N}\right)$. At each step it is essential to consistently keep all the terms $\delta^{(n)} \tilde{\mathcal{L}}_{2 k}\left(U^{(m)}, S_{2 m}\left(U^{(m)}\right)\right)$ with $N=k+m \times n$ where $U^{(m)}$ denotes the interpolating field after the $m$ th transformation. Only at the final iteration step is it allowed to simply perform the replacement according to Eq. (2.14). The reason is that the last transformation only removes terms which are proportional to the 'classical equation of motion' and modifies higher-order terms which one is not interested in. This is essentially the reason why the simple application of Eq. (2.14) to the $O\left(p^{4}\right)$ Lagrangian led to the correct answer up to $O\left(p^{4}\right)$.

We note the similarity of the algorithm to the Foldy-Wouthuysen method [17, 18] which is used to transform a Dirac Hamiltonian to a block-diagonal form up to a finite order $n$ in $1 / M$. It is well-known from this method that at each iteration step one consistently has to keep all terms up to order $n$ in the interaction Hamiltonian. This is required to generate S-matrix elements which are equivalent up to order $n$ in the $1 / M$ expansion. 


\section{SUMMARY}

We considered the use of field transformations for eliminating redundant structures in the framework of chiral perturbation theory. After stating the formal requirements for field transformations we derived the change in the functional form of the effective Lagrangian. In particular, we showed that for the purpose of identifying redundant terms, it is sufficient to make use of a simple replacement involving the 'classical equation of motion'. However, we explicitly demonstrated that this procedure is, in general, not suited to transform a given non-canonical Lagrangian into a canonical form. The reason is that it does not provide the quantitative change of the coefficients which multiply the independent structures. We described an iterative algorithm similar to the Foldy-Wouthuysen method which transforms a non-canonical into a canonical form up to any desired order in the momentum expansion.

\section{ACKNOWLEDGEMENTS}

This work was supported in part by a grant from the Natural Sciences and Engineering Research Council of Canada. One of the authors (S. S.) would like to thank A. Schaale, A. A. Bel'kov and A. V. Lanyov for stimulating discussions.

\section{APPENDIX A: TOY MODEL}

As an instructive example let us consider the following toy model [7]. The lowest-order Lagrangian $\mathcal{L}_{0}$ is taken to be the sum of two free Lagrangians of a massive scalar particle and a massless fermion, respectively,

$$
\mathcal{L}_{l}=\frac{1}{2}\left(\partial_{\mu} \Phi \partial^{\mu} \Phi-m^{2} \Phi^{2}\right)+i \bar{\Psi} \not \partial \Psi
$$

We introduce an effective interaction describing the decay of the scalar particle into a fermion-antifermion pair,

$$
\mathcal{L}_{e f f}=g_{e f f} \bar{\Psi} \Psi \square \Phi
$$

where the coupling constant $g_{\text {eff }}$ has the dimension energy ${ }^{-2}$. For example, with the above Lagrangian the invariant amplitude for fermion-fermion scattering, $A\left(p_{a}\right)+B\left(p_{b}\right) \rightarrow A\left(p_{a}^{\prime}\right)+$ $B\left(p_{b}^{\prime}\right)$, is found to be [7]

$$
M=-i g_{e f f}^{2}\left(\frac{t^{2}}{t-m^{2}}+\frac{u^{2}}{u-m^{2}}\right),
$$

where $t=\left(p_{a}-p_{a}^{\prime}\right)^{2}=\left(p_{b}-p_{b}^{\prime}\right)^{2}$ and $u=\left(p_{a}-p_{b}^{\prime}\right)^{2}=\left(p_{b}-p_{a}^{\prime}\right)^{2}$ are the Mandelstam variables 25]. Of course, the effective Lagrangian will contain interaction terms being of higher than first order in $g_{e f f}$, which may contribute to the scattering amplitude. However, for the point we want to make, a knowledge of the explicit form of such terms is not required.

Now let us consider the transformation method. If we introduce $\Phi=\chi+\delta \chi$, the Lagrangian may be written as 


$$
\begin{aligned}
\mathcal{L}(\Phi, \Psi)=\mathcal{L}^{\prime}(\chi, \Psi)= & \frac{1}{2}\left(\partial_{\mu} \chi \partial^{\mu} \chi-m^{2} \chi^{2}\right)+i \bar{\Psi} \not \partial \Psi-\delta \chi\left(\square+m^{2}\right) \chi \\
& -\frac{1}{2} \delta \chi \square \delta \chi-\frac{1}{2} m^{2}(\delta \chi)^{2}+g_{\text {eff }} \bar{\Psi} \Psi \square(\chi+\delta \chi),
\end{aligned}
$$

where we dropped an irrelevant total derivative. For the choice $\delta \chi=g_{\text {eff }} \bar{\Psi} \Psi$ the terms $-\delta \chi \square \chi$ and $g_{e f f} \bar{\Psi} \Psi \square \chi$ precisely cancel, and we are left with the Lagrangian

$$
\begin{aligned}
\mathcal{L}^{\prime}(\chi, \Psi)= & \mathcal{L}_{0}(\chi, \Psi)+\tilde{\mathcal{L}}_{e f f}(\chi, \Psi) \\
= & \frac{1}{2}\left(\partial_{\mu} \chi \partial^{\mu} \chi-m^{2} \chi^{2}\right)+i \bar{\Psi} \not \partial \Psi \\
& -m^{2} g_{\text {eff }} \bar{\Psi} \Psi \chi+\frac{1}{2} g_{\text {eff }}^{2} \bar{\Psi} \Psi\left(\square-m^{2}\right)(\bar{\Psi} \Psi) .
\end{aligned}
$$

The Lagrangian of Eq. (A5) is identical with the one obtained in Ref. [7] where, however, a different procedure has been used to derive a 'reduced' effective Lagrangian yielding the same on-shell matrix elements. finds

Clearly, for an effective interaction term, $\mathcal{L}_{\text {eff }}^{(2)}(\Phi)$, which is already of order $g_{\text {eff }}^{2}$, one

$$
\mathcal{L}_{\text {eff }}^{(2)}(\Phi)=\mathcal{L}_{\text {eff }}^{(2)}(\chi)+O\left(g_{\text {eff }}^{3}\right)
$$

and thus the same contribution results from such a term at order $g_{\text {eff }}^{2}$ in both representations. This is the reason why we did not explicitly specify the form of the higher-order Lagrangian.

If one wants to investigate processes at order $g_{\text {eff }}^{2}$, it is essential to consistently keep terms in the transformation up to that order. In order to see this we consider the scattering amplitude of two fermions at order $g_{\text {eff }}^{2}$ in the framework of Eq. (A5). The pole and contact diagrams yield, respectively,

$$
\begin{aligned}
& M_{P}=-i g_{\text {eff }}^{2}\left(\frac{m^{4}}{t-m^{2}}+\frac{m^{4}}{u-m^{2}}\right), \\
& M_{C}=-i g_{\text {eff }}^{2}\left(t+u+2 m^{2}\right),
\end{aligned}
$$

and the sum of the two contributions is the same as Eq. (A3). It is important to realize that the contact term results from two different sources. The first is a quadratic change in $\mathcal{L}_{0}$ and the second is a first order change in $\mathcal{L}_{\text {eff }}$. Only if one consistently keeps both terms, does one reproduce the correct answer.

A naive replacement in Eq. (A2) using the 'classical equation of motion' $(E O M)$ for $\Phi$ derived from $\mathcal{L}_{0}$,

$$
g_{e f f} \bar{\Psi} \Psi \square \Phi=g_{e f f} \bar{\Psi} \Psi\left(\square+m^{2}\right) \Phi-g_{e f f} m^{2} \bar{\Psi} \Psi \Phi \stackrel{E O M}{\rightarrow}-g_{e f f} m^{2} \bar{\Psi} \Psi \Phi,
$$

leads to incorrect results for S-matrix elements at order $g_{\text {eff }}^{2}$ since it does not generate the contact terms at order $g_{\text {eff }}^{2}$. One might argue that, in fact, one should use the equation of motion derived from $\mathcal{L}_{0}+\mathcal{L}_{e f f}$,

$$
\square \Phi=-m^{2} \Phi+g_{e f f} \square(\bar{\Psi} \Psi)
$$

to reexpress $\square \Phi$ in $\mathcal{L}_{e f f}$, and then work with the new Lagrangian. However, even though this method generates a contact term at order $g_{\text {eff }}^{2}$, it does not reproduce the correct S-matrix for fermion-fermion scattering as given by Eq. (A3). 


\section{REFERENCES}

[1] S. Weinberg, Physica 96A, 327 (1979).

[2] J. Gasser and H. Leutwyler, Ann. Phys. 158, 142 (1984).

[3] J. Gasser and H. Leutwyler, Nucl. Phys. B250, 465 (1985).

[4] J. Gasser, M.E. Sainio, A. Švarc, Nucl. Phys. B307, 779 (1988).

[5] S. Weinberg, Phys. Lett. B251, 288 (1990).

[6] S. Weinberg, Nucl. Phys. B363, 3 (1991).

[7] H. Simma, Z. Phys. C61, 67 (1994).

[8] Howard Georgi, Nucl. Phys. B361, 339 (1991).

[9] Christopher Arzt, preprint UM-TH-92-28 (1992), hep-ph/9304230.

[10] W. Buchmüller and D. Wyler, Nucl. Phys. B268, 621 (1986).

[11] Antonio Pich and Eduardo de Rafael, Nucl. Phys. B367, 313 (1991).

[12] H. Leutwyler, in Perspectives in the Standard Model, Proceedings of the 1991 Theoretical Advanced Study Institute in Elementary Particle Physics, Boulder, Colorado, 2 - 28 June 1991, ed. by R. K. Ellis, C. T. Hill and J. D. Lykken (World Scientific, Singapore, 1992).

[13] Carsten Grosse-Knetter, Phys. Rev. D49, 6709 (1994).

[14] R. Haag, Phys. Rev. 112, 669 (1958).

[15] S. Kamefuchi, L. O'Raifeartaigh and Abdus Salam, Nucl. Phys. 28, 529 (1961).

[16] S. Coleman, J. Wess and Bruno Zumino, Phys. Rev. 177, 2239 (1969).

[17] L. L. Foldy and S. A. Wouthuysen, Phys. Rev. 78, 29 (1950).

[18] H. W. Fearing, G. I. Poulis, S. Scherer, Nucl. Phys. A570, 657 (1994).

[19] T. E. Rudy, H. W. Fearing, and S. Scherer, Phys. Rev. C50, 447 (1994).

[20] John F. Donoghue, Eugene Golowich, Barry R. Holstein, Dynamics of the Standard Model (Cambridge University Press, Cambridge, 1992).

[21] Janos Balog, Phys. Lett. B149, 197 (1984).

[22] D. Ebert and H. Reinhardt, Nucl. Phys. B271, 188 (1986).

[23] R. D. Ball, Phys. Rep. 182, 1 (1989).

[24] D. Ebert, A. A. Bel'kov, A. V. Lanyov, A. Schaale, Int. J. Mod. Phys. A8, 1313 (1993).

[25] C. Itzykson and J.-B. Zuber, Quantum Field Theory (McGraw-Hill, New York, 1980). 